\title{
The Morphological Classification of the Penile Frenulum in Chinese's People and its Clinical Application Value in the Frenulum Malposition of Circumcision with the Disposable Circumcision Suture Device (DCSD)
}

Guang-Ming Liu ( $\sim$ brightlgm@126.com )

Tianjin First Center Hospital

Zi-Qiang Xu

Tianjin First Center Hospital

Research Article

Keywords: circumcision, circumcision device, disposable circumcision suture device, cosmetic result, patient satisfaction Introduction

Posted Date: October 14th, 2021

DOl: https://doi.org/10.21203/rs.3.rs-900176/v1

License: (1) This work is licensed under a Creative Commons Attribution 4.0 International License. Read Full License 


\section{Abstract}

Background: to conclude the clinical experience of the disposable circumcision suture device (DCSD), to describe the morphological classification of the penile frenulum in Chinese's people, to find a way to solve the malposition of the penile frenulum in the surgery with disposable circumcision suture device

Methods: From November 2013 to April 2021, there were 2265 consecutive Chinese patients with phimosis $367(16.2 \%)$ or redundant prepuce 1898 (83.8\%) who underwent circumcision in our hospital. Each patient's penile frenulum morphological feature was recorded and grouped according to the

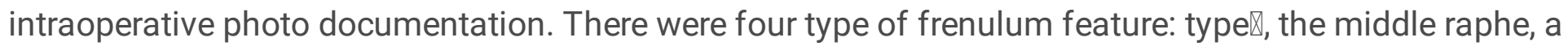
middle line longitudinal from the glan to the scrotal raphe; type , the middle double raphe or middle band; type $\varangle$, the diamond or lozenge-shaped raphe; type $\Downarrow$ was marked by the some hyperpigmentation zone. We also reported the convexity frenulum cutting site(CFCS) which could be palpated that a small rounded eminence tissue at the ventral prepuce middle raphe during the prodedure. Other data recorded were operation time, type of anesthesia, intra-operative blood loss, incision healing time, complications, mainly focus on the frenulum distortion, patient satisfaction.

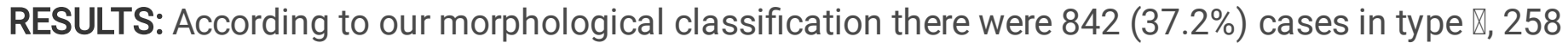

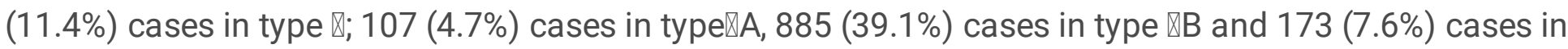

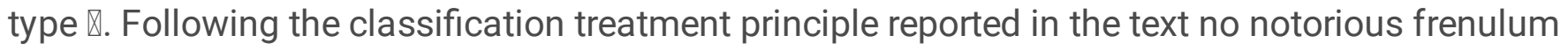
deviation was observed after the initial 100 cases.

Conclusion: The morphological classification of the penile frenulum incombinating with the anatomy marker of CFCS will facilitate accurate identification of the frenulum location, better define its right position, and provide a guide for the DCSD surgery for the individualized patients. The method described in this study could improve the success of the circumcision procedures, lower the related complication and is important to make the device acceptable and popular.

\section{Introduction}

Surgical Male circumcision (MC) is one of the most commonly used operative procedures worldwide for medical and traditional reasons[1]. Innovative approaches are required for the hospital to render better service to the patients[especially MC devices[2]. Most currently reported techniques can be categorized as dorsal slit, clamp, sleeve excision, and shield and suture less techniques. The disposable circumcision suture device (DCSD) or circumcision stapler, a novel device for circumcision, is the circular cutter with stapled anastomosis for circumcision[3]. It was applied clinically since 2012 reported by Yuan[4]. However, at the beginning of the procedure, we encountered so many complications such as bleeding, dehiscence, uncompleted cutting off the foreskin, frenulum distortion et al. The high rate of complication resulted from unfamiliar with or improper using the device application may distress the individuals, especially the frenulum distortion often resulting in the dissatisfaction of the patients. In traditional MC, the morphology of the frenulum was not paid attentio to, even was regarded of no clinical significance, 
for the sewing action was under direct view. Normally, no mismatching was encountered in most clinical traditional surgery setting. So no ideal observation and classification had yet been descripted or introduced. However, in DCSD, the frenulum wound was sewed with stapler closure, for the device design nature, this whole process, the cutting and sewing steps, can't been seen directly, frequently resulting the frenulum distortion. When this happening, the remedy method was to remove the suturing nail $3-4 \mathrm{~cm}$ near the penile frenulum and resew the wound with silk to correct the unsatisfied realignment of frenulum cut. If not, even if this does not affect the physiology function in most cases, it does affect the penis physical appearance and sexual psychological sometimes, both the patient and their sexual partner, occasionally induce the algopareunia[5]. We reported the morphological classification of the frenulum in Chinese individules. Based on this classification theory, we reported different management strategies in the DCSD surgery procedure accordingly. It is believed that the method could minimize related complication and optimize the aesthetic outcome during this procedure. After literature searching with' Medline, PUBMED' and other datebases, this is the first time to our knowledge to put forward the morphological classification of penile frenulum in chinese people, which was often initially ignored in MC and its application in clinical setting.

\section{Patients And Methods}

Clinical data

A non-randomized, prospective cohort study in which patients requesting DCSD device (brand LangHe) male circumcision, were enrolled in our hospital. From November 2013 to April 2021, there were 2265 consecutive Chinese patients (Han nation, 2089/2265, 92.2\%, others were minorities such as Man or Hui ehnic ) with phimosis $367(16.2 \%)$ or redundant prepuce 1898 (83.8\%) who underwent circumcision with DCSD performed by two experienced urologic doctors (the authors, who both having traditional surgical circumcision experience with more than 100 cases) in our hospital. The patients' ages range 8-55 years old (mean age $\pm S E: 31 \pm 4.7$ ); The medical indications for circumcision were personal hygiene $1876(82.8 \%)$, pain on erection for narrow ring $87(3.8 \%)$, preputial lesions $24(1.1 \%)$, therapeutic ( Balanitis) 207(9.1\%), Request by sexual parter $49(2.1 \%)$, others $22(0.1 \%)$. All participants were provided with information about the benefits and risks associated with the procedure, and were required to sign an informed consent form before being enrolled in the study. Patients with abnormalities of the genitalia such as penis dysplasia, concealed penis, or hypospadias, urinary tract infection, blood coagulation dysfunctions were all excluded from this study. Each patients' penile frenulum morphological feature was recorded and grouped according to the intraoperative photo documentation. Paraphimosis cases were treated with dorsal slitting and recovery for two weeks before circumcision for possible inflammation control. Routine blood tests were performed to exclude the acute infection cases. Participants were advised to abstain from sexual intercourse until the wound was completely healed and for at least eight weeks after the circumcision. Oral painkillers were recommended for postoperative pain. Patients were asked to keep the wound dry and clean by using sterile swabs after the first consultation. They were allowed to have shower 7 days later after the operation. Follow-up was at 2-3 days and four weeks. For those men who were not completely healed by four weeks, we conducted an additional six-week follow-up 
visit. They also were instructed to return sooner if they experienced bleeding, infection, or excessive pain. If the staplers were not fallen off successfully one months later, they were told to come back to removal the snails. We recorded the operation time, intra-operative blood loss, incision healing time, complications, mainly focus on the frenulum distortion, postoperative satisfaction.

\section{Anesthesia}

Two anesthesia methods were used for the procedure according to the patients'will and the ages. Some cases received needle-free anesthesia using topical lidocaine/prilocaine anesthetic, compound $5 \%$ lidocaine cream (Ziguang Pharmaceutical Co., Ltd. Beijing, China) alone. A volume of 2-5 ml of compound $5 \%$ lidocaine cream was evenly applied at the surface of the penis (including the glans and the penile body and root) 20-30 minutes before the operation. Other patients received a dorsal and circumferential penile block with 3-10 ml local anesthetic 1-2\% lidocaine. Some other cases received both.

The equipment and device

The equipment used in the DCSD procedure consisted of three forceps, one scissors, one needle holding (in case of suturing if needed) three Alice forceps. DCSD consists of glans bell with column, wing nut, strapping tape and handler(handle and shell)(Fig 1(1-2)). The bell and handler are available in type 36, 30,26 for adult correspond to different sizes of aduldts glans and type 18,15 to children. Device size used for including 30\# for 468 cases; $26 \#$ for 1399 cases, which were the most common sizes of chinese adult population; and 18\# or 15\# 398 cases which were usually used for Adolescence or child. Among them the $15 \#$ was usd in most of the younger than 12 years old. A specially designed meter was used to measure the circumference of the penis at the level of the coronal sulcus in order to select the appropriate device size. (Fig 1 (3)). The number of stainless steel staples depend the device size, there were $20,18,15,12$ to the size 30,26,18,15 accordingly which was most frequently used in Chinese people in our cases. The measurement of the glans was showed in Fig 1 (4). There already has a newer modified of DCSD, with a similar mechanical principle, except for the direction of force (Fig1(2)). However, for little clinic application with only couples of surgery in our unit, its clinical cases were not included within this study. The circumcision procedure see Fig 2.

The frenulum morphological classification

We presented the morphological classification of the frenulum and the penile raphe(Fig 3). It can be classified as the four type: typen, the middle raphe, just a middle line longitudinal from the glan to the scrotal raphe, it's the most frequent shape; typel,the middle double raphe or middle band; type $\square$, the diamond or lozenge-shaped raphe, were further characterized into the regular type $\square \mathrm{A}$ : the connection of the two corners longitudinal of the diamond is in straight line with the frenulum; if not, frequently deviation to one side would be classify to the unregular type $\mathrm{QB}$. In the type $\mathrm{GB}$ the diamond zone position could be anywhere along the middle line, with distal and proximal extending lines, generally close to the base of penis. The distal extending line of the lozenge-shaped configurate raphe deviated to either sides; 
type $\square$ was marked by the some hyperpigmentation zone, often run the length of the penis, no convex raphe was observed.

Distinctive type of the frenulum was treated with different strategy. The key point to make the frenulum meet perfect is to identify the convexity frenulum cutting site(CFCS) along lozenge-shaped. When fasten the glans bell囚it can be palpated that a small rounded eminence tissue at the ventral prepuce middle raphe crossing the surface of glans bell ring(as showing in Fig 4(4) red arrow). It was formed by the frenulum compressed to the glans bell under the pressure of the handler.We named it as CFCS (convexity frenulum at cutting site). These are the important reference markers in our method.

Avoiding penile frenulum misalignment was one of the character of a success circumcision. To achieve this goal, all procedure was conducted in accordance with the principles of alignment following 3 successive steps:

(1) the reference markers CFCS in red arrow was aiming at the scrotal raphe in yellow arrow as showed in Fig4(4), this is the first and most significant rule; (2) to fine adjust the the positon of outer prepuce at CFCS according the morphological classification of the frenulum, to make sure the positon of CFCS not moving during whole procedure next.

We explain in detail the exact procedures of the step 2 in our series further. As for type区, it was simple to

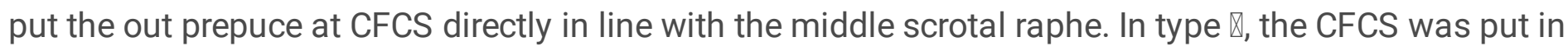

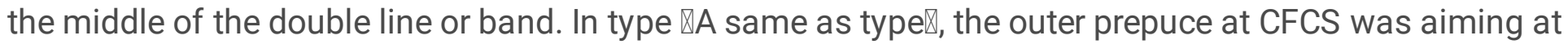
the scrotal raphe(See Fig4(1)). However in type $\triangle \mathrm{B}$, it was a little intricate. The skin at CFCS should be directed aligned to the base line of the scrotal raphe as far as possible, but if not achieveable, it should be compromised to keep the nature positon of the outlayer foreskin, in another word, to keep the CFCS and scrotal raphe in line. The inner layer of prepuce at CFCS must be fixed, only the overlying skin could be glid. The outlayer prepuce only played as a role of mild adjustment.

The judgment of success frenulum realignment

The perfect frenulum matching was defined as complete if 1 , The glans, frenulum, scrotal raphe kept sequentially in rows of one; 2 , whether erect or not, the frenulum and glans kept its natural position, without distortion or misregistration.

Placement of device and foreskin removal

We adopted a method of pressing to identify the proper length of foreskin removal in the flaccid state. The mons pubis was pressed to the symphysis pubis as possible in case of obesity or thick mons pubis substantial fat, to guarantee the proper foreskin was maintained for erection need(showed Fig 2). Once identify the cutting line expected, the incision line was marked outline $0.5 \mathrm{~cm}$ far away parallel with the coronal sulcus on the skin surface of the prepuce by mosquito clamps. The length of foreskin removal depended on some reference data, such as the patients' habius status; the substantial fat layer thickness with the mons pubis, the age and the degree of cutis laxa; inflammation and epidermis lesions, et al. The 
actual incision line was close near proximal to the mark line to remove the clamped marker. After disinfection and anesthesia, the prepuce was grasped at the 2, 6 and 10 o'clock positions using three forceps. Careful dissections bluntly or sharply were done in case of dense adhesion. The errhysis raw divided mucosa adhesions were smeared with erythromycin gel after surgery was over. The foreskin was retracted to expose the glans. In patients with phimosis, further antiseptic preparation of the glans was done after the dorsal slit was made. The glans bell of proper size chosen was placed to cover the corona, reassure the whole glans was entrapped to prevent the glans injury, then the prepuce was pulled over the glans bell with the three forceps above mentioned, strapping tape was used to fasten the foreskin to the column around the groove of the glans bell. For smaller type 15 model of the device, a purse-string suture was made instead of the strapping tape. The glans bell column was inserted through the middle of the handler, to adjust the angle to $30-45^{\circ}$ degree, to adjust the CFCS and the out layer prepuce according the theory above mentioned, next a wing nut was screwed to secured the glans bell and the handler. After removing the safety lock on the handler, the handle was squeezed and maintained for 10 seconds to trigger the internal circular scalpel blade and stapler for cutting and suture the foreskin. Then the wing nut was reverse screwed to release the handler and glans bell with the removal foreskin. The wound was checked for bleeding or dehiscence; additional suture with 2-0 silk was done if needed. The uncut tissue was dissected by scissors. The wound was bandaged with a gauze dressing with two layers of elastic bandage outside fasten; the latter was removed 2 , four hours separately after the surgery.

\section{Results}

The operation time were $11.4 \pm 3.9 \mathrm{~min}$. The volumes of intraoperative blood loss in our group was $2.2 \pm$ $1.4 \mathrm{ml}$. The staple-falling time was $17.6 \pm 3.7$ days. All cases were performed under local anesthesia, totally $501(22.1 \%)$ cases received topical anesthesia with compound $5 \%$ lidocaine cream. The proportion was especially high in children younger than 12 years old(137/175, 78.3\%), who refuse injective anesthesia in

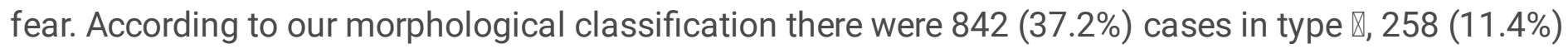

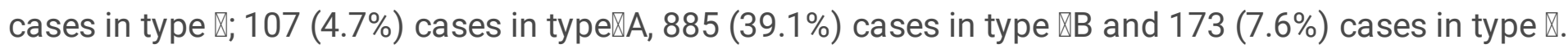
The morphlogical distribution was similar in the minorities such as Man or Hui ehnic population, with the type \and

$\llbracket \mathrm{B}$ were the majority $(132 / 176,75 \%)$.

Following the classification treatment principle no notorious frenulum deviation was observed after the initial 100 cases. However, there were 16 patients complained about their frenulum of prepuce malposition in the first 100 cases in our series. Four of them received frenulum cosmetics surgery.

Generally, most of the adverse events are mild and controllable, especially after finishing the learning curve following the standard operation protocols, about 100 cases later. The most common complication encountered after DSCD technique was the cyanosisy ecchymosis sign found on the ventral side of the glans. It is more apparent in that received injective anesthesia, mostly faded in two weeks. The other complications included residue nails longer than one months with a rate of 102 (4.5\%); foreskin edema, 
post-operative bleeds required suture $29(1.3 \%)$; post-operative dehiscence $24(1.0 \%) .94 .7 \%(2145 / 2265)$ of men were fully healed at four weeks of follow-up. The satisfactory rate was high $(92 / 100,92 \%)$, especially after the 100 cases later $(2163 / 2165,99.9 \%)$, when adopting our frenulum morphological classification. There were no devastating complications such as serious glans injury or urothelial injury.

\section{Discussion}

Worldwide circumcision prevalence rates are $30 \%$ of the total male population, making circumcision one of the most frequently performed surgical procedures throughout the world[6]. As an alternative to traditional surgery that required substantial training and experience, several circumcision devices have been evaluated to help achieve the goals of providing safe, cosmetic circumcision, including the DCSD[7]. The DCSD is a novel device based on bowel anastomotic stapler principles. Some studies have reported its initial application experience, including some comparing study and meta analysis[4,8-11]. The DCSD is unique in that it can be performed by a urologist himself on an outpatient basis, and no external device remains on the patient[4]. It also can be used in phimosis patients, which was precluded by some other instruments[12]. Being commercially attainable in 2012, as a novel surgery type, DCSD had just been undertaken in large clinical trials in recent three years in China and other countries[7]; no mentor was available from the beginning of DCSD clinic application in our hospital in 2013. Its poptimizeage and complication control world guarantee the success of the device application, also it be the key point if a new [13]device is accepted by the urology surgeon, practitioners, patients and their partners.

The morphological classification of the frenulum in chinese people was not reported previously as showed by our literature searching with' Medline, PUBMED' and other datebases. It was often initially ignored in traditional circumcision surgery for its little clinical significance and its limitation of application.

The frenulum malposition or distortion is a disappointing complication of circumcision using the DCSD, mostly occurring in early practice stage. Even a small proportion of the total people $(16 / 100,16 \%$ as showed in our 100 cases), it remained a real question about the emphasis of accurate frenulum realignment question. The malposition of the frenulum often frustrated the patients, impaired their sexual function or confidence, leading to the unsatisfying comment about the surgery outcome.

Unlike the ShangRing which could show the true frenulum keeping length, or the traditional circumcision, frenulum anastomosis was not under direct view in DCSD procedure, so the mild penis rotation inadvertently or adjusting of the cutting tissue length would alter the initial position of the anastomosis, especially in type $\triangle \mathrm{B}$ and $\nabla$ for the frenulum was not marked. At beginning we adopted a way of nature positioning, that is when the glans bell and handler closing to hold the penis in nature position, no dragging lateral of the skin, however, some frenulum malposition cases were fo post procedure. In case of malposition Salvage method was to remove3 to 4 suturing nails near the frenulum and resuture the slit edge to recover a relative right position. Frenulum presented with an array of morphological variations as showed in Fig 3. These morphological variations may sometimes pose a complicating factor in keep the 
optimal apposition in circumcision. Hence, appropriate recognition of frenulum variations and subsequent modification of treatment procedures are essential for a successful outcome of therapy. Some methods were proposed to identify the frenulum location with the scrotum and penile raphe, they mainly focus on the common morphologica type[14], that is equal to the type $₫$ or》 according to our classification. We do believe the method descriped by Huang was not suitable for all cases. After hundreds of observation we discribed the frenulum morphological classification system according to the frenulum and scrotal raphe appearance. (see Fig3). We proposed the different interventions according to the classification type. The morphological variations indeed presented in our clinical observations, but in conventional surgery it often was initially ignored for hand-sewn anastomosis of frenulum. It is well known that, the bilateral formation of the scrotum is indicated by the middle scrotal raphe, which continues anteriorly on the ventral surface of the penis as the penile raphe, and posteriorly along the median line of the perineum to the anus. This is the anatomy basis of frenulum classification theory. It should be gurantee that the penile raphe is connected with the distal frenulum. This is the key principle when handling this problem. Correctly understanding the morphological type and the strategy accordingly could solve the problem successfully. To our knowledge it is the first time to put forward the morphological type and its clinical significance, Not only meaningful to the DCSD but also to other circumcision procedures such as the Shang Ring .After we adopted this methods, no obvious frenulum distortion was observed in our series any more. This method will make the glan frenulum and the base of penis in line in either penile erection or flaccid state. The morphological classification of the frenulum and the strategy accordingly could maximally decrease the danger of mismatch of frenulum. Remember this could avoid almost all of the frenulum mislocation situation in clinical practice.[14]

The frenulum morphological description was also suitable for the child in our serious. However, as the penis was Incomplete developed for the child, it is hard to identify the obvious anatomical markings such as the scrotal raphe, but the marker of convexity frenulum cutting site(CFCS) could be noticed in almost all the cases. It is helpful for the child circumcision. Young boys less than eight years was not included in our series for extra sedation and anesthesia intervention must be given. For most younger patients (aged less than 8 years old) it had to be performed at a hospital under general anaesthetic. As our data showed most of the child 175 less than 12 years old successfully received the DCSD procedures without severe post-operative complications. There were some experiences, the DCSD smaller than type 15 not fit for the using of trapping tape for the small glans bell, it should be used the silk suture instead. The glans of children were more delicate and soft vulnerable to injury, so it is important to confirm all the glan tissues were completely trapped in the bell before triggering the device. It is not recommended to start the DCSD procedure in children unless rich experience was accumulated.

The main limitation in this study is only the Chinese people mainly Han nation's dates were collected, only including a few of other minorities nation. Further study including vary of ethnic, and age distributions will identify its effective value and verify its applicabitlity. Other limitation was lacking of a randomized controlled trial provides more convincing in its clinical application. The findings reported here thus provide a foundation for further studies which, preferably, will include the completion of a randomized controlled trial. 


\section{Conclusion}

The morphological classification of the penile frenulum can be classified as the four type with different morphologic features. We also reported the convexity frenulum cutting site(CFCS) which could be palpated that a small rounded eminence tissue at the ventral prepuce middle raphe. The application of this classification of the frenulum incombinating with the anatomy CFCS point will facilitate accurate identification of the frenulum location, better define its right position, and provide a guide for the DCSD surgery for the individualized patients.

These DCSD procedures are acceptable for its convenience, single-man manipulated and almost suture less[10]. The method described in this study could improve the success of the circumcision procedures, lower the related complication and is important to make the device acceptable and popular.

\section{Declarations}

- Ethics approval and consent to participate : Compliance with ethical standards: All procedures performed in studies involving human participants were in accordance with the ethical standards of the institutional and/or national research committee and with the 1964 Helsinki declaration and its later amendments or comparable ethical standards. All participants were provided with information about the benefits and risks associated with the procedure, and were required to sign an informed consent form before being enrolled in the study. The study was approved by Tianjin First Center Hospital clinical research ethics committee.

- Consent for publication: Not applicable.

- Availability of data and materials: All data generated or analysed during this study are included in this published article!

- Competing interests: The authors declare that they have no competing interests

- Funding: Not applicable

- Authors' contributions: Guang-Ming Liu concluded the morphological feature of the penile frenulum and was a major contributor in writing the manuscript. Zi-Qiang Xu analyzed and interpreted the patient data regarding the circumcision patients. All authors read and approved the final manuscript.

- Acknowledgements: the authors appreciated DoctorWei Wang, Professor Qian Liu and Professor Hong-Shun ma for their supporting on the clinical work. The authors would like to acknowledge all the patients who participated in the study and the dedicated providers.

\section{References}

1 Ndagijimana A, Mugenzi P, Thomson DR, Hedt-Gauthier B, Condo JU, Ngoga E. PrePex Male Circumcision: Follow-Up and Outcomes during the First Two Years of Implementation at the Rwanda Military Hospital. PLoS One 2015; 10: e0138287 
2 Lebina L, Milovanovic M, Otwombe K et al. PrePex circumcision surveillance: Adverse events and analgesia for device removal. PLoS One 2018; 13: e0194271

3 Senel FM, Demirelli M, Oztek S. Minimally invasive circumcision with a novel plastic clamp technique: a review of 7,500 cases. Pediatr Surg Int 2010; 26: 739-45

4 Yuan Y, Zhang Z, Cui W et al. Clinical investigation of a novel surgical device for circumcision. J Urol 2014; 191: 1411-5

5 Czajkowski M, Czajkowska K, Zarańska K et al. Male Circumcision Due to Phimosis as the Procedure That Is Not Only Relieving Clinical Symptoms of Phimosis But Also Improves the Quality of Sexual Life. Sex Med 2021; 9: 100315

6 Bronselaer GA, Schober JM, Meyer-Bahlburg HF et al. Male circumcision decreases penile sensitivity as measured in a large cohort. BJU Int 2013; 111: 820-7

7 Pozza D, Pozza C, Mosca A, Pozza M. Preputial circumcision performed with a new mechanical stapling tool. The "langhe disposable circumcision suture device". Preliminary experiences. Arch Ital Urol Androl 2020; 91: 261-2

8 Huo ZC, Liu G, Li XY et al. Use of a disposable circumcision suture device versus conventional circumcision: a systematic review and meta-analysis. Asian J Androl 2017; 19: 362-7

9 Huo ZC, Liu G, Li XY et al. Use of a disposable circumcision suture device versus conventional circumcision: a systematic review and meta-analysis. Asian J Androl 2017; 19: 362-7

10 Zhang Z, Yang B, Yu W et al. Application of a novel disposable suture device in circumcision: a prospective non-randomized controlled study. Int Urol Nephrol 2016; 48: 465-73

11 Lv BD, Zhang SG, Zhu XW et al. Disposable circumcision suture device: clinical effect and patient satisfaction. Asian J Androl 2014; 16: 453-6

12 Millard PS, Goldstuck ND. No-needle, single-visit adult male circumcision with Unicirc: a multi-centre field trial. PLoS One 2015; 10: e0121686

13 Jiang W, Fu JL, Guo WL et al. A Modified Pressure Dressing to Avoid Severe Bleeding After Circumcision With a Disposable Circumcision Suture Device and a Discussion on the Mechanism of Bleeding With the Disposable Circumcision Suture Device. Sex Med 2021; 9: 100288

14 Huang YQ, Zheng J, Zhou XX et al. [Frenulum identification positioning with a disposable suture device in circumcision to prevent postoperative penile frenulum malposition]. Zhonghua Nan Ke Xue 2017; 23: 422-6

\section{Figures}


2

\section{Figure 1}

showed the schematic of the circumcision device.

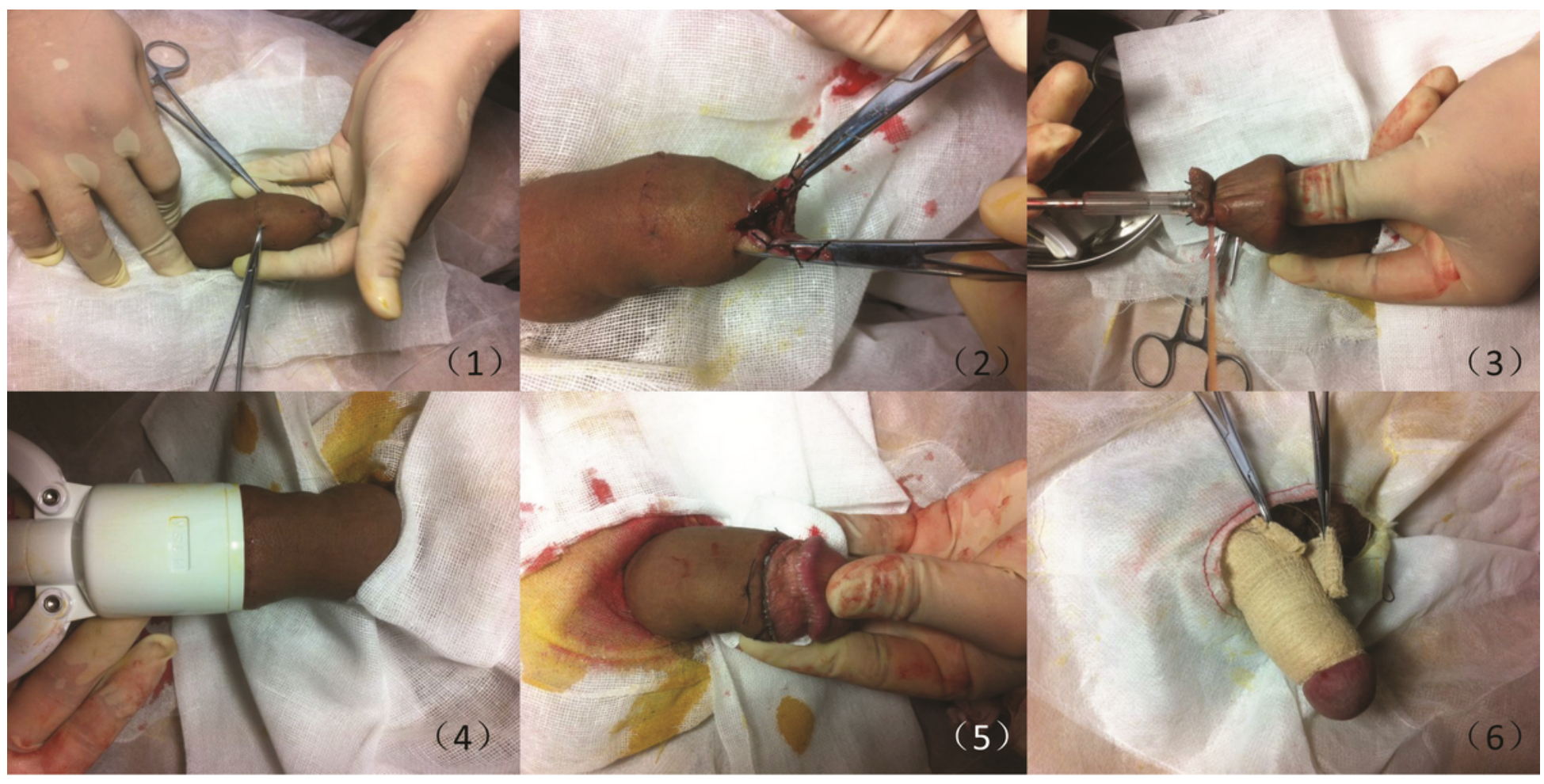

Figure 2

(1) to (6) shows the whole procedure 


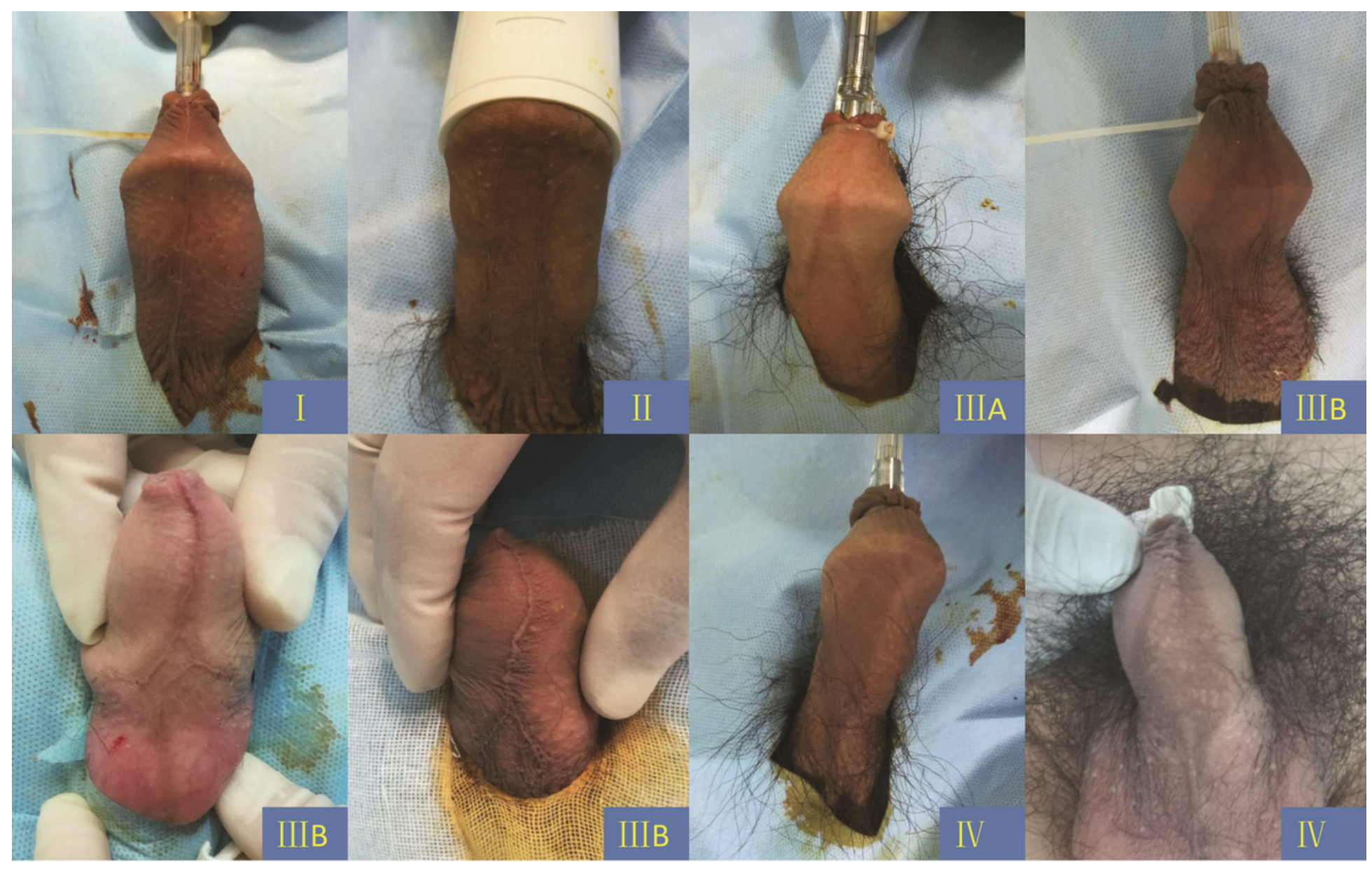

Figure 3

(1) to (8) showing morphological classification of the frenulum and the penile raphe 


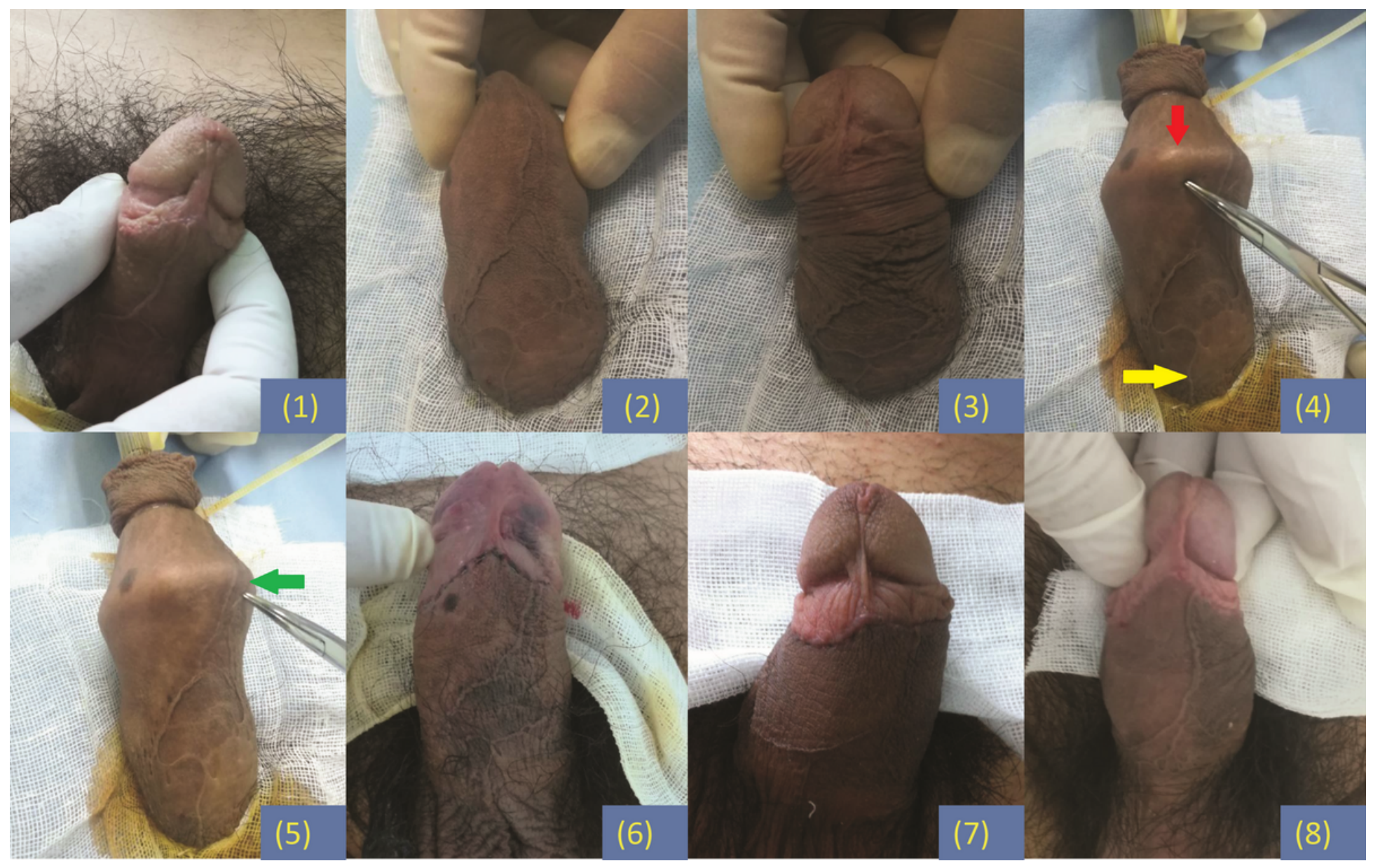

\section{Figure 4}

showing the frenulum in Alignment in type $₫ A$ and $₫ B$. (1)the type $₫ A$ after operation. (2) Neutral appearance of the penis; (3) Retraction of the prepuce exposes the glans ; (4) forceps pointing to the convexity frenulum cutting site in red arrow, ie.CFCS, noting the distal extending line of the lozengeshaped raphe deviated to right side (left to the patient himself). (5) forceps pointing to intersection of the distal extending line and the glans bell ring (in green arrow), if this site is forced to be connected instead of CFCS with the line of scrotal raphe, it will result in distortion indeed, indeed that is the wrong direction. (6) Two days after DCSD surgery showing the right frenulum position. In the Fig4 (2) to (6) was all the same patient. (7-8) other examples of the irregular diamond type of frenulum type $\mathbb{B}$. 